\title{
Decomposition of Convex Figures into Similar Pieces
}

\author{
M. Laczkovich \\ Department of Analysis, Eötvös Loránd University, \\ Budapest, Múzeum krt 6-8, Hungary 1088
}

Communicated by Imre Bárány

\begin{abstract}
If a convex plane figure $P$ can be decomposed into finitely many nonoverlapping convex figures such that one of these pieces is similar to $P$, then $P$ is a polygon. Also, if $P$ can be decomposed into infinitely many nonoverlapping sets such that each of the pieces is similar to $P$, then $P$ is a polygon.
\end{abstract}

By a convex figure we mean a compact convex subset of the plane with nonempty interior. The following statement is mentioned, without proof, on p. 1 of [1].

If a convex figure $P$ is the union of finitely many (but at least two) nonoverlapping and congruent sets similar to $P$, then $P$ is a polygon.

In this note we prove the following two generalizations of this result.

Theorem 1. Suppose that the convex figure $P$ is the union of finitely many (but at least two) nonoverlapping convex figures such that one of them is similar to $P$. Then $P$ is a polygon.

Theorem 2. Suppose that the convex figure $P$ is the union of infinitely many nonoverlapping sets similar to $P$. Then $P$ is a polygon.

We use the following notation. The diameter, interior, closure, boundary, and derived set (set of points of accumulation) of a set $A$ are denoted by $\operatorname{diam} A$, int $A$, cl $A, \partial A$, and $A^{\prime}$. If $A$ is convex, then we denote the set of extremal points of $A$ by $E(A)$. The isolated points of $E(A)$ are called vertices, and the set of vertices of $A$ is denoted by $V(A)$. It is easy to see that $p \in V(A)$ if and only if $p$ is the common endpoint of two nonparallel segments contained in $\partial A$. The angle of these segments 
is denoted by $\alpha_{A}(p)$. It is clear that $V(A)$ is always countable and for every $\varepsilon>0$ the set $\left\{p \in V(A): \alpha_{A}(p)<\pi-\varepsilon\right\}$ is finite. Therefore, if $V(A)$ is infinite and $p_{1}, p_{2}, \ldots$ is an enumeration of $V(A)$, then $\alpha_{A}\left(p_{n}\right) \rightarrow \pi$.

We denote the set $(E(A))^{\prime}$ by $K(A)$. Thus $E(A)=V(A) \cup K(A)$. It is easy to see that $p \in \partial A \backslash K(A)$ if and only if there is an open subarc $I$ of $\partial A$ such that $p \in I$ and $I$ consists of at most two line segments. Clearly, $K(A)=\emptyset$ if and only if $A$ is a polygon.

To prove Theorem 1, suppose that $P$ is a convex figure, $P=\cup_{i=1}^{n} P_{i}$, where $P_{1}, \ldots, P_{n}$ are nonoverlapping convex figures and $P_{1}$ is similar to $P$. Then

$$
\partial P_{1} \cap \text { int } P \subset \bigcup_{i=2}^{n}\left(P_{1} \cap P_{i}\right) .
$$

Indeed, every $x \in \partial P_{1} \cap$ int $P$ is a point of accumulation of $\bigcup_{i=2}^{n} P_{i}$. Thus $x \in$ $P_{1} \cap P_{i}^{\prime} \subset P_{1} \cap P_{i}$ for at least one $i \geq 2$. Since the sets $P_{1} \cap P_{i}$ are line segments (or points), it follows from (1) that $\partial P_{1} \cap$ int $P$ can be covered by finitely many lines. Therefore the next lemma yields Theorem 1 .

Lemma 1. Let $P$ and $P_{1} \subsetneq P$ be similar convex figures such that $\partial P_{1} \cap$ int $P$ can be covered by finitely many lines. Then $P$ is a polygon.

Proof. First we note that $E\left(P_{1}\right) \cap$ int $P$ is finite, as $E\left(P_{1}\right)$ intersects every line in at most two points. Let $\phi$ denote the similarity transformation mapping $P$ onto $P_{1}$. We show that $\phi(K(P)) \subset K(P)$. Let $p \in K(P)$ and $p_{1}=\phi(p)$. Obviously, $p_{1} \in$ $K\left(P_{1}\right)$ and hence $p_{1} \in E\left(P_{1}\right)^{\prime}$. Since $E\left(P_{1}\right) \cap$ int $P$ is finite, it follows that $p_{1} \in$ $\left(E\left(P_{1}\right) \cap \partial P\right)^{\prime}$. We claim that $p_{1} \in K(P)$. If this is not true, then $p_{1} \in \partial P \backslash E(P)$ or $p \in V(P)$. In both cases there are two line segments, $I$ and $J$, in $\partial P$ having $p_{1}$ as a common endpoint. Since $p_{1} \in\left(E\left(P_{1}\right) \cap \partial P\right)^{\prime}$, one of these line segment, say $I$, contains at least two distinct elements $q \neq p_{1}$ and $r \neq p_{1}$ of $E\left(P_{1}\right) \cap \partial P$. Then $p_{1}$, $q$, and $r$ are collinear points of $\partial P_{1}$ and hence there is a line segment $I^{\prime} \subset I \cap \partial P_{1}$ containing $p_{1}, q$, and $r$. However, in this case $q$ and $r$ cannot be both extremal points of $P_{1}$, a contradiction. Therefore $p_{1} \in K(P)$ as we stated.

Consequently, $K(P)$ is a compact subset of $\partial P$ that is mapped into itself by the contraction $\phi$. If $P$ is not a polygon, then $K(P) \neq \emptyset$ and it follows that $\phi$ has a fixed point $p_{0} \in K(P)$. Let $S$ denote the angular domain between the half-tangents of $P$ at $p_{0}$ and containing $P$ ( $S$ is a half-plane if $P$ has a tangent at $p_{0}$ ). Since $\phi(P)=P_{1} \subset P$, it is easy to see that $\phi(S)=S$. This implies that either $\phi$ is a homothetic transformation or it is a homothetic transformation followed by a reflection. In both cases, $q, \phi^{2}(q)$, and $p_{0}$ are collinear for every point $q$.

Since $p_{0} \in K(P)$, we have $p_{0}=\phi\left(p_{0}\right) \in K\left(P_{1}\right)=E\left(P_{1}\right)^{\prime}$. Since $E\left(P_{1}\right) \cap$ int $P$ is finite, it follows that $p_{0} \in\left(E\left(P_{1}\right) \cap \partial P\right)^{\prime}$. This implies, in particular, that $E\left(P_{1}\right) \cap$ $\partial P$ is infinite. Let $q_{1} \in E\left(P_{1}\right) \cap \partial P$ be arbitrary, and put $q=\phi^{-1}\left(q_{1}\right)$ and $q_{2}=$ $\phi\left(q_{1}\right)$. Then $q \in E(P) \subset \partial P$ and $q_{2} \in \partial P_{1}$, since $q_{1} \in \partial P$. As we remarked above, the points $q, q_{2}$, and $p_{0}$ are collinear. Now we distinguish between two cases. If $q_{2} \in \partial P$, then $q, q_{2}$, and $p_{0}$ are collinear points of $\partial P$ and thus the segment $I$ with endpoints $q$ and $p_{0}$ belongs to $\partial P$. In this case $q_{1}=\phi^{-1}\left(q_{2}\right) \in \phi^{-1}(I)$. Clearly, there are at most two lines that intersect $\partial P$ in a segment with endpoint $p_{0}$, and 
hence those points $q_{1}$ of $E\left(P_{1}\right) \cap \partial P$ for which $q_{2} \in \partial P$, can be covered by at most two lines.

If, on the other hand, $q_{2} \in$ int $P$, then $q_{1}=\phi^{-1}\left(q_{2}\right) \in \phi^{-1}\left(\partial P_{1} \cap\right.$ int $\left.P\right)$. Since, by assumption, $\partial P_{1} \cap$ int $P$ can be covered by finitely many lines, we obtain that those points $q_{1}$ of $E\left(P_{1}\right) \cap \partial P$ for which $q_{2} \notin \partial P$, can be covered by finitely many lines. Summing up, $E\left(P_{1}\right) \cap \partial P$ can be covered by finitely many lines. However, $E\left(P_{1}\right)$ contains at most two elements of every line and hence $E\left(P_{1}\right) \cap \partial P$ must be finite. This contradicts our previous statement that $E\left(P_{1}\right) \cap \partial P$ is infinite. Therefore $K(P)=\emptyset$; that is, $P$ is a polygon.

Now we turn to the proof of Theorem 2, and suppose that $P=\cup_{i=1}^{\infty} P_{i}$, where $P_{1}, P_{2}, \ldots$ are nonoverlapping sets similar to $P$. We say that a point $p$ is critical, if every neighborhood of $p$ intersects infinitely many of the sets $P_{i}$.

Lemma 2. Let $G \subset P$ be open and suppose that $G$ contains no critical points. Then $E\left(P_{i}\right) \cap G$ is finite for every $i$.

Proof. If $p \in\left(\partial P_{i}\right) \cap G$, then there is a neighborhood $U$ of $p$ that intersects only a finite number of the sets $P_{j}$. This easily implies that every point of $\left(\partial P_{i}\right) \cap U$ is contained in a $P_{j}$ with some $j \neq i$ (see the proof of (1)), and hence $\left(\partial P_{i}\right) \cap U$ is covered by finitely many sets of the form $P_{i} \cap P_{j}(i \neq j)$. Since these sets are line segments (or points), it follows that $E\left(P_{i}\right) \cap U$ is finite, and hence $E\left(P_{i}\right) \cap G=$ $V\left(P_{i}\right) \cap G$ for every $i$.

Suppose that $E\left(P_{i}\right) \cap G=V\left(P_{i}\right) \cap G$ is infinite, and let $p_{1}, p_{2}, \ldots$ be an enumeration of $V(P)$. As we remarked earlier, we have $\alpha_{P}\left(p_{n}\right) \rightarrow \pi(n \rightarrow \infty)$, where $\alpha_{P}\left(p_{n}\right)$ denotes the angle of the two line segments of $\partial P$ with endpoint $p_{n}$. If $p \in V\left(P_{i}\right) \cap G$, then there is a neighborhood of $p$ that intersects only a finite number of the sets $P_{j}$ such that $p$ is a common vertex of these $P_{j}$ 's. If the angles at these vertices are $\alpha_{P}\left(p_{n_{i}}\right)(i=1, \ldots, k)$, then $\alpha_{P}\left(p_{n_{1}}\right)+\cdots+\alpha_{P}\left(p_{n_{k}}\right)=v \pi$, where $v=1$ or 2 (we have $v=1$ if $p \in \partial P_{j} \backslash E\left(P_{j}\right)$ for some $j$ ). Since $\alpha_{P}\left(p_{n}\right)>0$ and $\alpha_{P}\left(p_{n}\right) \rightarrow \pi$, it is easy to see that the number of sets of indices $\left\{n_{1}, \ldots, n_{k}\right\}$ satisfying these equations is finite. However, if $V\left(P_{i}\right) \cap G$ is infinite, then infinitely many different angles must occur among these indices (namely, the angles of $P_{i}$ at the points of $\left.V\left(P_{i}\right) \cap G\right)$, which is a contradiction.

Lemma 3. Suppose that $P$ is not a polygon. Let $G \subset P$ be an open set containing a critical point and let $n$ be a positive integer. Then there is a closed disk $D \subset G \backslash P_{n}$ such that int $D$ also contains a critical point.

Proof. First we assume that $G \backslash P_{n}$ does not contain critical points. This implies, by Lemma 2, that $E\left(P_{i}\right) \cap\left(G \backslash P_{n}\right)$ is finite for every $i$. If $i \neq n$, then $E\left(P_{i}\right) \cap P_{n}$ is also finite, since $P_{i}$ and $P_{n}$ are nonoverlapping convex sets. That is, $E\left(P_{i}\right) \cap G$ is finite for every $i \neq n$.

Since the sets $P_{i}$ are similar and are contained in $P$, it follows that diam $P_{i} \rightarrow 0$. As every neighborhood of a critical point $p \in G$ intersects infinitely many $P_{i}$ 's, it 
follows that $G$ contains infinitely many of the sets $P_{i}$. If $P_{i} \subset G$ and $i \neq n$, then $E\left(P_{i}\right)$ is finite; that is, $P_{i}$ is a polygon. Then so is $P$, contradicting our assumption.

Therefore $G \backslash P_{n}$ must contain at least one critical point $p$. If $D$ is a small closed disk around $p$ such that $D \cap P_{n}=\emptyset$, then $D$ satisfies the requirements of the lemma.

Now we prove Theorem 2 assuming that int $P$ contains a critical point. If $P$ is not a polygon, then, by Lemma 3, there is a closed disk $D_{1} \subset$ int $P$ such that $D_{1} \cap P_{1}=\emptyset$ and int $D_{1}$ contains a critical point. Applying Lemma 3 again, we can choose a closed disk $D_{2} \subset$ int $D_{1}$ such that $D_{2} \cap P_{2}=\emptyset$ and int $D_{2}$ contains a critical point. Continuing this process, we can define the nested sequence of closed disks $D_{1} \supset$ $D_{2} \supset \cdots$. If $p \in \cap_{n=1}^{\infty} D_{n}$, then $p \in P$ and $p \notin \bigcup_{n=1}^{\infty} P_{n}$, a contradiction.

Therefore, in order to prove Theorem 2, we may assume that int $P$ contains no critical points. In this case, by Lemma 2 , the sets $V_{i}=E\left(P_{i}\right) \cap$ int $P$ are finite for every $i$.

Our next aim is to show that, for at least one $i, \partial P_{i} \cap$ int $P$ can be covered by finitely many lines. For a given $i$, consider the components of the set $\partial P_{i} \backslash\left(\partial P \cup V_{i}\right)$. These components are open subarcs of $\partial P_{i}$, and, as they do not contain extremal points of $P_{i}$, they are line segments. These line segments are of two kinds: either their endpoints are in $\partial P$; that is, they are chords of $P$, or at least one of their endpoints is in int $P$. In the latter case these endpoints belong to $V_{i}$ and hence the number of these line segments is finite (at most twice the cardinality of $V_{i}$ ). That is, if $\partial P_{i} \cap$ int $P$ cannot be covered by finitely many lines, then $\partial P_{i}$ contains infinitely many chords of $P$. It is easy to see that in this case $\partial P \backslash P_{i}$ has infinitely many components.

Suppose this happens for every $i$. Then $\partial P \backslash P_{1}$ has infinitely many components; let $I_{1}$ be a component with diam $I_{1}<1$. Clearly, $I_{1}$ is an open subarc of $\partial P$. Let $P_{i_{2}} \cap I_{1} \neq \emptyset$; then, as $P_{1}$ and $P_{i_{2}}$ are nonoverlapping convex sets, it follows that $P_{i_{2}}$ is contained in the convex hull of $I_{1}$. This implies that each component of $\partial P \backslash P_{i_{2}}$, with at most one exception, is also a component of $I_{1} \backslash P_{i_{2}}$. Let $I_{2}$ be one of these components with $\mathrm{cl} I_{2} \subset I_{1}$ and $\operatorname{diam} I_{2}<1 / 2$. Let $P_{i_{3}} \cap I_{2} \neq \emptyset$; then $P_{i_{3}}$ is contained in the convex hull of $I_{2}$. Thus every component of $\partial P \backslash P_{i_{3}}$, except one, is also a component of $I_{2} \backslash P_{i_{3}}$; let $I_{3}$ be one of these components with $\mathrm{cl} I_{3} \subset I_{2}$ and diam $I_{3}<1 / 3$. In this way we can define the nested sequence of subarcs $I_{1} \supset$ $I_{2} \supset \cdots$. If $p \in \cap_{n=1}^{\infty} \mathrm{cl} I_{n}$, then $p$ cannot be covered by any of the sets $P_{i}$, which is impossible.

Therefore at least one of the sets $\partial P_{i} \cap$ int $P$ must be contained in a finite system of lines. Then an application of Lemma 1 completes the proof of Theorem 2.

Problems and Remarks. 1. The analogue of Theorem 1 in higher dimension is not true: every cone $C$ can be decomposed into two nonoverlapping convex sets such that one of them is similar to $C$. In connection with this example, T. Zamfirescu asked the following (personal communication, June 1994);

Let $P$ be a convex figure in $\mathbf{R}^{3}$ and suppose that $P$ is the union of finitely many nonoverlapping convex figures such that two of the pieces are similar to $P$. Does this imply that $P$ is a polyhedron? 


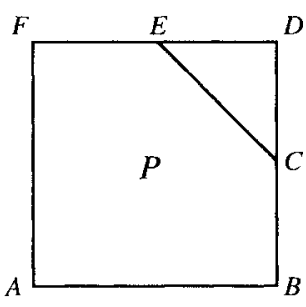

Fig. 1

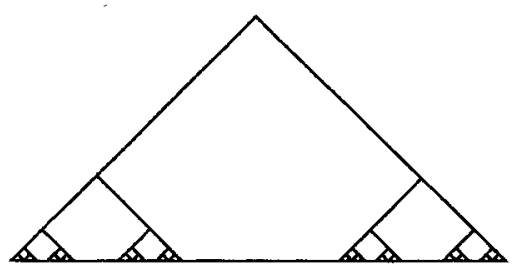

Fig. 2

2. We do not know whether or not the higher dimension analogue of Theorem 2 is true.

3. Not every convex polygon $P$ can be decomposed into infinitely many nonoverlapping sets similar to $P$. A necessary condition for the existence of such a decomposition is that $2 \pi$ is a linear combination of the angles of $P$ with nonnegative integer coefficients. Indeed, suppose that this condition is not satisfied, and, still, there is a decomposition $P=\cup_{n=1}^{\infty} P_{n}$ with the given properties. This easily implies that whenever $p \in$ int $P$ is the vertex of any of the polygons $P_{n}$, then each neighborhood of $p$ contains infinitely many polygons $P_{k}$. Then we can find a sequence of closed disks $D_{n}$ such that int $P \supset D_{1} \supset D_{2} \supset \ldots$, the center of each $D_{n}$ is a vertex of one of the polygons $P_{k}$, and $D_{n} \cap P_{n}=\emptyset$ for every $n$. If $p \in \cap_{n=1}^{\infty} D_{n}$, then $p \in P \backslash \cup_{n=1}^{\infty} P_{n}$ which is impossible.

This necessary condition is not sufficient. For example, it can be shown that the regular hexagon cannot be decomposed into infinitely many nonoverlapping regular hexagons.

4. It is easy to prove that if a polygon $P$ can be decomposed into finitely many nonoverlapping sets similar to $P$, then it can also be decomposed into infinitely

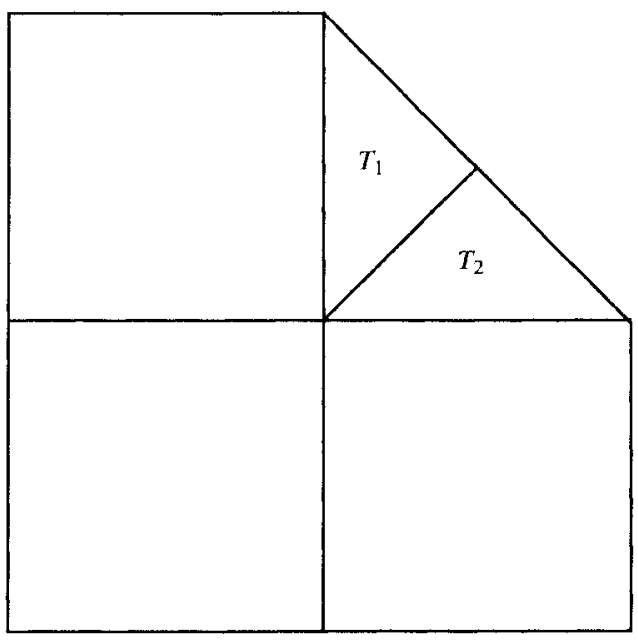

Fig. 3 
many such sets. The converse, however, is not true, as the following simple example shows.

Let $P$ denote the pentagon with vertices $A B C E F$ on Fig. 1 , where $A B D F$ is a square and $C$ and $E$ are the middle points of the sides $B D$ and $D F$. It is clear that $P$ cannot be decomposed into finitely many nonoverlapping sets similar to $P$. For the sake of brevity we say that a set $S$ has an infinite $P$-tiling if $S$ can be decomposed into infinitely many nonoverlapping sets similar to $P$. We want to show that $P$ has an infinite $P$-tiling.

(i) As Fig. 2 shows, removing a Cantor-set from the hypotenuse of an equilateral right triangle, the remaining set has an infinite $P$-tiling.

(ii) Then it follows that every square has an infinite $P$-tiling (see Fig. 1).

(iii) Finally, Fig. 3 shows an infinite $P$-tiling of $P$ itself; the sets $T_{1}$ and $T_{2}$ have infinite $P$-tilings by (i), and the remaining three squares have infinite $P$-tilings by (ii).

\section{Reference}

1. G. Valette and T. Zamfirescu, Les partages d'un polygone convexe en 4 polygones semblambes au premier, J. Combin. Theory Ser. B 16 (1974), 1-16.

Received July 14, 1994, and in revised form August 29, 1994. 